\section{Transcatheter tricuspid valve edge-to-edge repair for severe tricuspid regurgitation in a Chinese patient}

An 80-year-old man with recurrent right heart failure despite optimal heart failure therapy, was referred to our institution. He had inferior myocardial infarction, had undergone a coronary artery bypass graft operation and had a permanent pacemaker.

Echocardiography showed a dilated right ventricle with impaired systolic function (Tricuspid annular plane systolic excursion (TAPSE) $10 \mathrm{~mm}$ ), a dilated tricuspid annulus (septal-lateral diameter $4 \mathrm{~cm}$ ) and massive $(4+)$ functional tricuspid regurgitation (TR) (figure 1A). The tricuspid valve (TV) was structurally intact without impingement or perforation by the pacemaker lead (figure $2 \mathrm{~A}, \mathrm{~B}$ ). The heart team did not consider him fit for open repair because of the previous sternotomy and his frailty (score 6/9), ${ }^{1}$ and recommended percutaneous edge-to-edge TV repair with the MitraClip device.

The procedure was performed, without any complications, under general anaesthesia with interventional guidance by transoesophageal echocardiography and fluoroscopy (figure 2). The first and the second clips were positioned at the anteroseptal commissure (figure 2C) and the posteroseptal commissure, respectively, resulting in moderate $(2+)$ TR (figure $2 \mathrm{H}$ ), and mean transvalvular gradient $3 \mathrm{~mm} \mathrm{Hg}$. At 1-month follow-up, the patient reported symptomatic improvement from New York Heart Association class IV to class II, and echocardiography showed two clips at TV with moderate TR, and improvement in hepatic vein reversal (figure 1D,H).

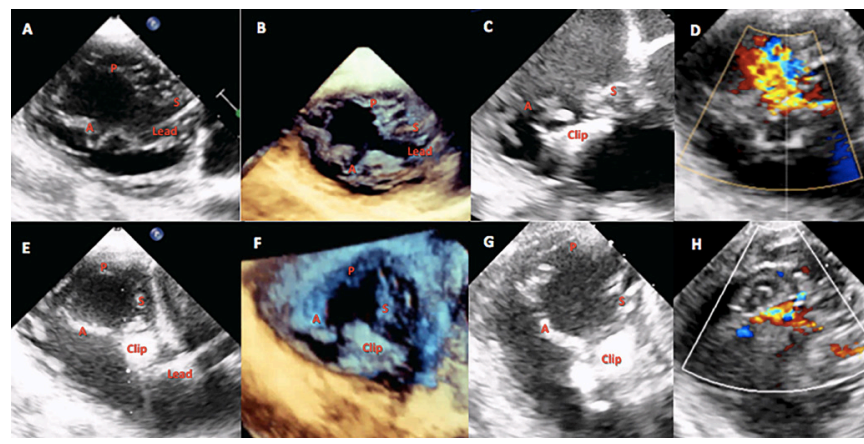

Figure 2 (A) Transgastric view of 2D TOE prior to tricuspid valve (TV) clipping: pacing lead was located at the anteroseptal commissure without leaflet impingement. (B) Transgastric view of 3D TOE prior to TV clipping: this allowed us to appreciate the relationship between the TV leaflet and the pacing lead. (C) Grasping view using mid-oesophageal view of 2D TOE: both anterior and septal leaflets were successfully grasped by the clip. (D) Colour flow Doppler of transgastric view of TV: severe tricuspid regurgitation (TR) as demonstrated by a wide vena contracta area. (E) Transgastric view of 2D TOE after TV clipping: both anterior and septal leaflets were inserted, pacing lead remained at anteroseptal commissure. (F) Transgastric view of 3D TOE right after TV clipping: doubly confirmed the leaflet insertion. (G) Grasping view using transgastric view by 2D TOE: it confirmed the grasping was secured. $(\mathrm{H})$ Colour flow Doppler of transgastric view of TV: mild-to-moderate TR after application of the second clip at the posteroseptal commissure. 2D, two-dimensional; 3D, three dimensional; $A$, anterior leaflet of TV; $\mathrm{S}$, septal leaflet of TV; $\mathrm{P}$, posterior leaflet of TV; Lead, right ventricular pacing lead; TOE, transoesophageal echocardiography.

Transcatheter treatment of severe TR with the edge-to-edge MitraClip technique has been reported in predominantly Caucasian populations. ${ }^{23}$ There is racial difference in right heart structure and function that may affect effectiveness of transcatheter intervention

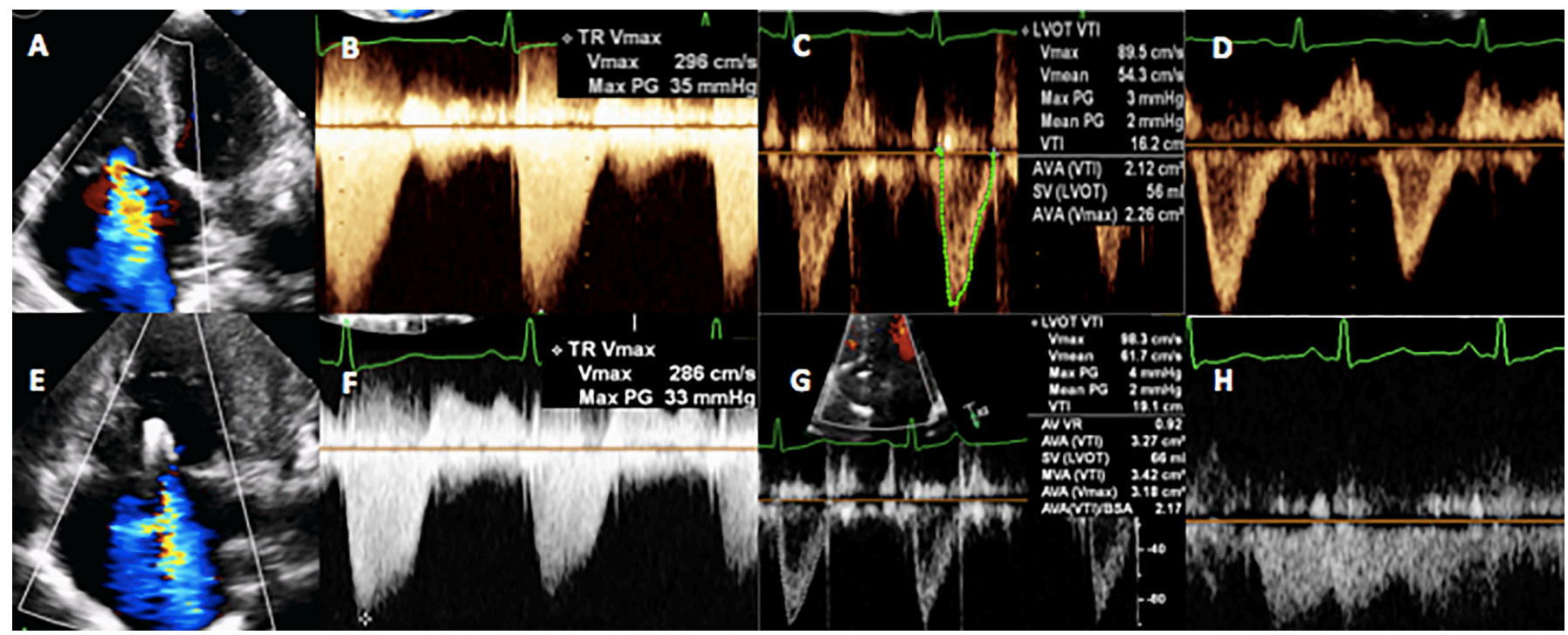

Figure 1 (A) Colour flow Doppler of tricuspid valve (TV) from the apical four-chamber view: massive tricuspid regurgitation (TR) with an effective regurgitation orifice (ERO) was $0.35 \mathrm{~cm}^{2}$. (B) Continuous wave Doppler of TR: dense early peaking TR signal with no evidence of significant pulmonary hypertension. (C) Pulse wave Doppler at left ventricular outflow tract (LVOT) level: LVOT velocity time integral (VTI) was moderately reduced due to significant TR. (D) Pulse wave Doppler at the hepatic vein: late systolic flow reversal which was consistent with significant TR. (E) Colour flow Doppler of TV from the apical four-chamber view: TR has been decreased to mild-to-moderate severity, ERO was only $0.24 \mathrm{~cm}^{2}$. (F) Continuous wave Doppler of TR: no change in pulmonary pressure. (G) Pulse wave Doppler at LVOT level: LVOT VTI was significantly improved to $19.1 \mathrm{~cm}$ which translated into $18 \%$ increase in stroke volume. (H) Pulse wave Doppler at the hepatic vein: mainly systolic forward flow without any reversal. 
in Asian patients. ${ }^{4}$ To the best of our knowledge, this is the first report of MitraClip treatment for TR in Asia. We demonstrated the potential feasibility, safety and effectiveness of this technique in Chinese patients.

\section{Gary S H Cheung, ${ }^{1}$ Kevin K H Kam, ${ }^{1}$ Yat-yin Lam, ${ }^{2}$ Alex P W Lee ${ }^{1}$}

${ }^{1}$ Division of Cardiology, Department of Medicine and Therapeutics, Faculty of Medicine, The Chinese University of Hong Kong and Prince of Wales Hospital, Hong Kong, China

${ }^{2}$ Center Medical, Hong Kong, Hong Kong

Correspondence to Dr Gary S H Cheung, Division of Cardiology, Department of Medicine and Therapeutics, Clinical Sciences Building, Prince of Wales Hospital, Shatin, Hong Kong, China; garycsh@hotmail.com

Contributors GSHC and KKHK collected and analysed the data and drafted the manuscript. APWL and Y-yL revised the manuscript critically and approved the final version for publication.

Funding The authors received no financial support for the research, authorship and/or publication of this article.

Competing interests APWL received speaker honorarium from Abbott and Philips Healthcare.

Patient consent Parental/guardian consent obtained.
Ethics approval Chief of Service of Department of Medicine \& Therapeutics, Prince of Wales Hospital, Hong Kong; Chairman, Central Committee of Cardiology, Hospital Authority, Hong Kong.

Provenance and peer review Not commissioned; externally peer reviewed.

(C) Article author(s) (or their employer(s) unless otherwise stated in the text of the article) 2018. All rights reserved. No commercial use is permitted unless otherwise expressly granted.

\section{D) Check for updates}

To cite Cheung GSH, Kam KKH, Lam Y, et al. Heart Asia 2018;10:e010997.

Heart Asia 2018;10:e010997. doi:10.1136/heartasia-2017-010997

\section{REFERENCES}

1. Clinical Frailty Scale. Canadian Study on Health \& Aging, Revised, 2008.

2. Hammerstingl C, Schueler R, Malasa M, et al. Transcatheter treatment of severe tricuspid regurgitation with the MitraClip system. Eur Heart J 2016;37:849-53.

3. Nickenig G, Kowalski M, Hausleiter J, et al. Transcatheter treatment of severe tricuspid regurgitation with the edge-to-edge mitraclip technique. Circulation 2017;135:1802-14.

4. Kawut SM, Lima JA, Barr RG, et al. Sex and race differences in right ventricular structure and function: the multi-ethnic study of atherosclerosis-right ventricle study. Circulation 2011;123:2542-51. 